\title{
Perfil metabólico de riesgo cardiovascular en niños y adolescentes obesos con menor sensibilidad insulínica
}

\author{
Raquel Burrows $A^{1}$, Medardo Burgueño $A^{1}$, Laura Leiva $B^{2 a}$, \\ Ximena Ceballos $S^{1 b}$, Ivette Guillier $0^{1}$, Vivien Gattas $Z^{2 b}$, \\ Lidia Lera $M^{1 c}$, Cecilia Albala $B^{1}$. \\ Cardiovascular risk and metabolic \\ profile in obese children and \\ adolescents with low insulin \\ sensitivity
}

Background: Infantile obesity is associated with metabolic disturbances (hiperinsulinism, impaired glucose, dislypidemia) that determine a higher risk of type 2 diabetes, high blood pressure and atherosclerotic vascular disease in adulthood. Insulin resistance is a central mechanism of complications of obesity and is associated to body fat mass. Aim: To investigate insulin sensitivity and its association with anthropometric and metabolic variables in obese children. Patients and methods: We studied 314 children aged 6 to 15 years. Of these, 235 had a body mass index (BMI) over the 95 percentile for age and sex (classified as obese) and 79 had a normal body mass index; 161 were pre-pubescent and 153 were pubescent. Weight, height, percentage of total body fat (\% TBF) using the sum of 4 skinfolds, blood pressure (BP), waist circumference (WC), basal glucose (Glu) and insulin (Ins) were measured. Insulin sensitivity (IS) was calculated with the homeostasis model assessment (HOMA) and QUICKI index. Serum lipids, postprandial Glu and Ins were measured only in obese children. Results: BMI, WC and \% TBF had an inverse and significant correlation with basal IS ( $p<0.001$ ). Obese children had higher BP, basal Ins, and HOMA and a lower QUICKI index, compared to normal weight children. A low basal IS was present in $58 \%$ of obese children. Obese children with low IS had higher WC, \% TBF, triglycerides, total/HDL-cholesterol ratio, basal and 2-h post glucose Gli and Ins and lower HDL-cholesterol than obese children with normal IS. In prepubescent children, the risk of a low IS was 2.43 times higher with a TBF over $33 \%$. In pubescent children, it was 2.92 times higher with a TBF over $37 \%$. Conclusions: Low IS in prepubescent and pubescent obese children is associated with central obesity and a higher cardiovascular risk (Rev Méd Chile 2005; 133: 795-804).

(Key Words: Body mass index; Homeostasis; Insulin resistance; Obesity)

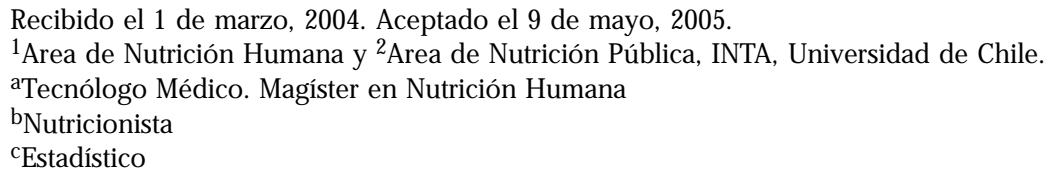

Correspondencia a: Raquel Burrows A. José Pedro Alessandri 5540, Macul. Fax: 2214030. E-mail: rburrows@inta.cl 
L a obesidad infantil ha sido considerada por la Organización Mundial de la Salud (OMS) "una enfermedad crónica", porque se perpetúa en el tiempo y se asocia a un síndrome metabólico de resistencia insulínica (SMRI), que determina a futuro un mayor riesgo de diabetes mellitus tipo 2 (DM2), hipertensión arterial (HA) y enfermedades cardiovasculares isquémicas (ECVI) ${ }^{1,2}$. La relación entre la resistencia insulínica (RI), el sobrepeso y el mayor riesgo cardiovascular, está dada porque al compromiso del metabolismo glucídico se agrega el lipídico y una disfunción endotelial temprana y progresiva, que constituye la base del daño ateroesclerótico que caracteriza a estas enfermedades ${ }^{3-6}$.

El aumento de la grasa corporal, especialmente abdominal, la etnicidad y la presentación de la pubertad, han sido identificados en población menor de 20 años como contribuyentes a este SMRI ${ }^{7}$. Los diferentes estudios longitudinales coinciden en que el riesgo de presentar estas enfermedades crónicas, tiene como base una susceptibilidad étnico-genética para desarrollar una insulinorresistencia, cuya expresión está estrechamente asociada a la obesidad centrípeta, a la dieta rica en grasa y al sedentarismo $8-10$

La descendencia de los pueblos nativos americanos muestra una mayor prevalencia de RI y DM 2 , que llega a $50 \%$ a los 35 años en la población de indios Pimas expuesta a los estilos de vida occidentales ${ }^{11-13}$. Chile se caracteriza por una gran proporción de habitantes de etnia aborigen, la que llega a casi $70 \%$ en población urbana ${ }^{14}$. Diferentes estudios nacionales de tipo transversal coinciden en la alta prevalencia de hipercolesterolemia e hiperinsulinismo basal y postglucosa en población infantil obesa $(27 \%, 42,2 \%$ y 63\%, respectivamente) $)^{15}$. El objetivo de este estudio fue investigar el perfil metabólico (glucídico y lipídico) y la presión arterial, según grado de sensibilidad insulínica en niños obesos y la asociación de la insulinosensibilidad basal con algunas variables biológicas (sexo, pubertad y grasa corporal). Dada la alta exposición a los factores ambientales (dieta rica en grasas saturadas y azúcares y sedentarismo) propios del modelo de desarrollo económico que ha tenido el país en la última década, identificar en nuestra población algunos determinantes de la expresión de la RI y focalizar al grupo de obesos con mayor riesgo cardiovascular, contribuirá a la optimización de los recursos para la prevención de las enfermedades crónicas no transmisibles del adulto.

\section{MATERIAL Y MÉTODO}

Estudio transversal que incluyó una muestra de 235 niños obesos (IMC >p95 del referente CDCNCHS) ${ }^{16}$ de ambos sexos, 123 prepúberes y 112 púberes, entre 6 y 15 años, que consultaron al Centro Diagnóstico del INTA (CEDINTA) entre los años 2000 y 2003 y una muestra de 79 niños eutróficos (IMC entre p10 y 85 del NCHS-CDC) de ambos sexos, 38 prepúberes y 41 púberes entre 6 y 15 años, seleccionados de un estudio realizado en el mismo centro, entre los años 2001 y 2003, para determinar niveles de insulina y glicemia en población infantil chilena (muestra de normales). Ambos estudios fueron aprobados por el Comité de Etica del INTA, solicitándose el consentimiento firmado de uno de los padres o tutores.

Antropometría. En cada niño se realizó, al ingreso, un examen físico que incluyó las siguientes evaluaciones: IMC, midiendo el peso y la talla con una balanza electrónica de precisión (SECA) con cartabón incluido de acuerdo a normas internacionales (metodología de Frankfurt). El porcentaje de grasa corporal total (GCT) por sumatoria de 4 pliegues cutáneos (bicipital, tricipital, subescapular y suprailíaco, medidos con cáliper de Lange) utilizando las ecuaciones de Deurenberg y Westrate ${ }^{17,18}$. La distribución de grasa por el perímetro de la cintura (a la altura del ombligo) con huincha no distensible de fijación automática (SECA). El grado de pubertad por los 5 estadios de Tanner, considerando el desarrollo mamario en la mujer y el genital en el varón y calificando como prepúberes a los niños en etapa 1 de Tanner. La presión arterial diastólica (PAD) y sistólica (PAS) con un esfigmomanómetro de mercurio con manguito ad hoc, utilizando la metodología estandarizada por el "Second task force" sobre control de presión arterial en niños del National Heart, Lung and Blood Institute ${ }^{19}$.

Laboratorio. En todos los niños se evaluó la glicemia e insulinemia basal, para lo cual se 
extrajeron $5 \mathrm{ml}$ de sangre venosa en ayunas. La glicemia por método enzimático colorimétrico GOD-PAP (Química Clínica Aplicada S.A.) y la insulinemia por radioinmunoanálisis (RIA Diagnostic Products Corporation). La sensibilidad insulínica basal se calculó a través de los Indice de Sensibilidad Insulínica de QUICKI ${ }^{20,21}$ (1/(log glicemia ayuno $(\mathrm{mg} / \mathrm{dl})+\mathrm{Log}$ insulina ayuno (uUI/ $\mathrm{ml})$ ) y $\mathrm{HOMA}^{22}$ (insulina ayuno (uUI/dl) * glicemia ayuno (mmol/lt)/22,5). En los niños obesos, se evaluó además, el perfil de colesterol (colesterol total, colesterol LDL, colesterol HDL y triglicéridos), mediante metodología analítica seca (Vitros, Johnson \& Johnson, Clinical diagnostics Inc) y la glicemia e insulinemia postsobrecarga oral de glucosa $(1,75 \mathrm{~g} / \mathrm{kg}$ de peso con un máximo de 75 g), para lo cual se extrajo $5 \mathrm{ml}$ de sangre venosa 2 $\mathrm{h}$ después de la ingestión de glucosa.

Estadística. Los resultados se analizaron según grado de desarrollo puberal (prepúberes y púberes), separando la muestra de niños de acuerdo a su adecuación ponderal en eutróficos y obesos. Los niños obesos fueron comparados, además, de acuerdo a la sensibilidad insulínica basal. Se consideró que existía una sensibilidad insulínica disminuida si el índice de QUICKI era $\leq 0,34$ en los prepúberes $\mathrm{y} \leq 0,32$ en los púberes, ya que estos valores corresponden al tercil más bajo de QUICKI, de un referente nacional elaborado en una muestra de 225 niños sanos de 6 a 15 años 23 .

Las variables antropométricas, la presión arterial, así como las que determinan el perfil lipídico y glucídico se describen con las medias y desviación estándar. La asociación entre algunas variables antropométricas y la sensibilidad insulínica se determinaron con el coeficiente de correlación de Pearson. Se utilizó t de Student para comparar los promedios entre 2 grupos y por tablas de contingencia simple, se calculó el riesgo por Odds Ratio $\mathrm{y}$ las asociaciones entre variables por el $\mathrm{chi}^{2}$. Se estableció un $\mathrm{p}<0,05$, el punto de corte para la significancia estadística.

\section{RESULTADOS}

La Tabla 1 muestra algunas características biológicas y antropométricas según estado nutricional.

Tabla 1. C aracterísticas generales de la muestra según estado nutricional

\begin{tabular}{|lcccc|}
\hline & \multicolumn{2}{c}{ Prepúberes } & \multicolumn{2}{c|}{ Púberes } \\
& Eutróficos & Obesos & Eutróficos & Obesos \\
& $(38)$ & $(123)$ & $(41)$ & $(112)$ \\
\hline Edad (años) & $8,4 \pm 1,9^{a}$ & $8,8 \pm 1,8$ & $12,2 \pm 1,7$ & $12,2 \pm 1,7$ \\
Sexo (hombre/mujer) & $20 / 18$ & $67 / 56$ & $15 / 26$ & $40 / 72$ \\
IMC $^{\text {b }}$ (ptje Z) & $0,3 \pm 0,6$ & $4,1 \pm 1,5^{* * *}$ & $0,3 \pm 0,9$ & $3,6 \pm 1,3^{* * *}$ \\
PAS $^{\text {( }}$ & $102,2 \pm 11,5$ & $103,2 \pm 14,0$ & $105,0 \pm 11,9$ & $117,4 \pm 15,1^{* * *}$ \\
PAD $^{\text {d }}$ & $59,0 \pm 5,5$ & $65,4 \pm 9,4^{* *}$ & $60,5 \pm 9,2$ & $71,8 \pm 10,0^{* * *}$ \\
Perímetro cintura (cm) & $59,1 \pm 6,4$ & $82,8 \pm 10,3^{* * *}$ & $66,2 \pm 7,7$ & $96,9 \pm 12,1^{* * *}$ \\
GCTe (\%) & $13,0 \pm 5,5$ & $31,9 \pm 4,8^{* * *}$ & $21,6 \pm 6,3$ & $36,6 \pm 4,4^{* * *}$ \\
Glicemia basal (mg/dl) & $85,0 \pm 8,7$ & $85,5 \pm 9,1$ & $85,6 \pm 8,1$ & $86,2 \pm 8,6$ \\
Insulinemia basal (uUI/dl) & $5,9 \pm 7,8$ & $11,7 \pm 7,1^{* *}$ & $10,5 \pm 13,2$ & $17,8 \pm 10,5^{* * *}$ \\
ISI QUICKI & $0,40 \pm 0,04$ & $0,34 \pm 0,03^{* * *}$ & $0,37 \pm 0,06$ & $0,32 \pm 0,03^{* * *}$ \\
ISI HOMA & $1,1 \pm 0,9$ & $2,5 \pm 1,5^{* *}$ & $2,2 \pm 2,6$ & $3,8 \pm 2,3^{* *}$ \\
\hline
\end{tabular}

aPromedio \pm desviación estándar

bIndice de masa corporal

cPresión arterial sistólica

dPresión arterial diastólica

eGrasa corporal total

Significancia según t de student: ${ }^{*} \mathrm{p}<0,05 ;{ }^{*} \mathrm{p}<0,01$; ${ }^{* * *} \mathrm{p}<0,000$. 
Tanto en prepúberes como en púberes, no se observaron diferencias en los promedios de la edad y en las glicemias de ayuno al comparar eutróficos y obesos. Los obesos mostraron promedios significativamente mayores en todas las variables antropométricas, en la presión arterial diastólica, en la insulinemia de ayuno y en el índice HOMA y menor promedio en el índice de QUICKI al compararlos con los eutróficos. En los púberes, se observó, además, mayores promedios de presión arterial sistólica en los obesos.
Tanto en púberes como en prepúberes, hubo una correlación inversa y significativa $(p<0,000)$ entre la sensibilidad insulínica basal y el IMC, el perímetro de cintura y el porcentaje GCT (Tabla 2).

La Tabla 3 muestra las características generales y antropométricas de los obesos, según la sensibilidad insulínica basal evaluada por el índice de QUICKI. Tanto en prepúberes como en púberes, no hubo diferencias en la edad ni en la presión arterial al comparar los obesos insulinosensibles con los obesos de menor sensibilidad. En ambos

Tabla 2. C oeficiente de correlación entre variables antropométricas y sensibilidad insulínica (QUICKI)

\begin{tabular}{|lcccccccc|}
\hline & Varones prepúberes & \multicolumn{2}{c|}{ Mujeres prepúberes } & \multicolumn{2}{c|}{ Varones púberes } & \multicolumn{2}{c|}{ Mujeres púberes } \\
\hline Variables & $\mathrm{r}^{*}$ & $\mathrm{p}^{* *}$ & $\mathrm{r}^{*}$ & $\mathrm{p}^{* *}$ & $\mathrm{r}^{*}$ & $\mathrm{p}^{* *}$ & $\mathrm{r}^{*}$ & $\mathrm{p}^{* *}$ \\
IMC $\left(\mathrm{kg} / \mathrm{m}^{2}\right)$ & $-0,572$ & $<0,000$ & $-0,418$ & $<0,000$ & $-0,572$ & $<0,000$ & $-0,418$ & $<0,000$ \\
Perímetro de cintura $(\mathrm{cm})$ & $-0,539$ & $<0,000$ & $-0,490$ & $<0,000$ & $-0,539$ & $<0,000$ & $-0,490$ & $<0,000$ \\
$\%$ GCT & $-0,576$ & $<0,000$ & $-0,399$ & $<0,000$ & $-0,576$ & $<0,000$ & $-0,399$ & $<0,000$ \\
\hline
\end{tabular}

${ }^{*}$ Coeficiente de correlación de Pearson. ${ }^{* *}$ Significancia según t de Student.

Tabla 3. C aracterísticas generales y antropometría de la muestra de obesos según sensibilidad insulínica (SI) basal

\begin{tabular}{|lcccc|}
\hline & \multicolumn{2}{c}{ Prepúberes } & \multicolumn{2}{c|}{ Púberes } \\
& $\begin{array}{c}\text { SI basal } \\
\text { normal } \\
(54)\end{array}$ & $\begin{array}{c}\text { SI basal } \\
\text { disminuida } \\
(69)\end{array}$ & $\begin{array}{c}\text { SI basal } \\
\text { normal } \\
(45)\end{array}$ & $\begin{array}{c}\text { SI basal } \\
\text { disminuida } \\
(67)\end{array}$ \\
\hline Edad (años) & $8,8 \pm 1,6^{\mathrm{a}}$ & $8,9 \pm 2,0$ & $12,1 \pm 1,7$ & $12,4 \pm 1,7$ \\
Sexo (hombre/mujer) & $30 / 24$ & $39 / 30$ & $21 / 24$ & $19 / 48$ \\
IMC $^{\mathrm{b}}$ (ptje Z) & $3,9 \pm 1,3$ & $4,4 \pm 1,6$ & $3,3 \pm 1,0$ & $3,9 \pm 1,3^{* *}$ \\
PAS $^{\text {(p) }}$ & $102,7 \pm 17,1$ & $103,2 \pm 12,0$ & $114,5 \pm 14,9$ & $119,3 \pm 14,9$ \\
PAD $^{\text {d }}$ & $65,9 \pm 9,9$ & $65,0 \pm 9,8$ & $71,7 \pm 9,0$ & $73,2 \pm 10,0$ \\
Perímetro cintura (cm) $_{\text {GCT }}$ (\%) & $81,9 \pm 8,8$ & $85,6 \pm 9,7^{*}$ & $93,4 \pm 10,3$ & $100,5 \pm 12,3^{* * *}$ \\
& $30,8 \pm 4,4$ & $33,6 \pm 4,2^{* *}$ & $35,3 \pm 4,0$ & $37,6 \pm 4,2^{* * *}$ \\
\hline
\end{tabular}

aPromedio \pm desviación estándar

bIndice de masa corporal

cPresión arterial sistólica

dPresión arterial diastólica

eGrasa corporal total

Significancia según t de student: ${ }^{*} p<0,05 ;{ }^{* *}<<0,025 ;{ }^{* * *} p<0,01$. 
Tabla 4. Perfil lípidico y glucídico en niños obesos según sensibilidad insulínica (SI) basal

\begin{tabular}{|c|c|c|c|c|}
\hline & \multicolumn{2}{|c|}{ Prepúberes } & \multicolumn{2}{|c|}{ Púberes } \\
\hline & $\begin{array}{l}\text { SI basal } \\
\text { normal } \\
(54)\end{array}$ & $\begin{array}{c}\text { SI basal } \\
\text { disminuida } \\
\text { (69) }\end{array}$ & $\begin{array}{c}\text { SI basal } \\
\text { normal } \\
(45)\end{array}$ & $\begin{array}{c}\text { SI basal } \\
\text { disminuida } \\
(67)\end{array}$ \\
\hline $\mathrm{CT}(\mathrm{mg} / \mathrm{dl})^{\mathrm{b}}$ & $178,4 \pm 31,3^{a}$ & $183,2 \pm 31,3$ & $176,9 \pm 34,3$ & $172,7 \pm 40,4$ \\
\hline Col-LDL $(\mathrm{mg} / \mathrm{dl})^{\mathrm{C}}$ & $111,6 \pm 26,1$ & $112,6 \pm 25,7$ & $112,2 \pm 30,6$ & $104,5 \pm 37,3$ \\
\hline Col-HDL (mg/dl) $)^{d}$ & $48,4 \pm 11,4$ & $44,3 \pm 11,7^{*}$ & $46,6 \pm 7,7$ & $43,0 \pm 9,7^{*}$ \\
\hline Triglicéridos (mg/dl) & $94,8 \pm 51,8$ & $121,3 \pm 65,7^{* *}$ & $93,0 \pm 50,7$ & $124,1 \pm 60,1^{\text {*** }}$ \\
\hline CT/Col-HDL & $3,8 \pm 1,0$ & $4,3 \pm 1,0^{*}$ & $3,8 \pm 0,9$ & $4,2 \pm 1,3^{*}$ \\
\hline Glicemia basal (mg/dl) & $82,9 \pm 8,2$ & $86,3 \pm 8,8^{*}$ & $83,8 \pm 8,5$ & $88,2 \pm 8,1^{\text {*w* }}$ \\
\hline Insulinemia basal (uUI/dl) & $6,4 \pm 2,2$ & $16,2 \pm 6,6^{\text {*adk }}$ & $9,6 \pm 3,3$ & $24,5 \pm 9,6^{* \text { *atk }}$ \\
\hline ISI QUICKI & $0,37 \pm 0,04$ & $0,34 \pm 0,03^{* 4 * 4}$ & $0,35 \pm 0,02$ & $0,30 \pm 0,01^{* 4+k}$ \\
\hline ISI HOMA & $1,3 \pm 2,0$ & $3.4+1.4^{* 40 \times k}$ & $2,0 \pm 0.6$ & $5.3 \pm 2.0^{* * 1+2 k}$ \\
\hline Glicemia $2 \mathrm{~h}(\mathrm{mg} / \mathrm{dl})$ & $89,2 \pm 14,5$ & $94,9 \pm 17,9^{*}$ & $92,2 \pm 11,4$ & $98,7 \pm 14,7^{* * *}$ \\
\hline Insulina $2 \mathrm{~h}(\mathrm{uUI} / \mathrm{dl})$ & $36,5 \pm 20,6$ & $68,5 \pm 53,4^{\text {*⿻丷木火火 }}$ & $48,6 \pm 30,0$ & $107,1 \pm 79,5^{\text {**ak }}$ \\
\hline
\end{tabular}

aPromedio \pm desviación estándar

${ }^{b}$ Colesterol total

${ }^{\mathrm{c} C o l e s t e r o l ~ L D L}$

${ }^{\mathrm{d} C o l e s t e r o l ~ H D L}$

eSignificancia según $\mathrm{t}$ de student

${ }^{*} \mathrm{p}<0,05 ;{ }^{* *} \mathrm{p}<0,025 ;{ }^{* * * *} \mathrm{p}<0,01 ;{ }^{* * * * *} \mathrm{p}<0,000$.

grupos puberales, los obesos con menor sensibilidad insulínica tenían mayores promedios de perímetro de cintura y porcentaje GCT que los de mayor sensibilidad insulínica, con diferencias significativas en el IMC sólo en los púberes.

La Tabla 4 muestra los perfiles metabólicos en los niños obesos según la insulinosensibilidad basal. Tanto en púberes como en prepúberes, los obesos con menor sensibilidad insulínica muestran promedios significativamente mayores de triglicéridos, índice CT/colHDL, glicemia e insulinemia de ayuno y $2 \mathrm{~h}$ postglucosa y menores promedios de colesterol HDL que los obesos con mayor sensibilidad insulínica.

$\mathrm{Al}$ analizar la asociación de algunas variables biológicas con la sensibilidad insulínica en la población de niños obesos, no se observó asociación significativa con la presencia de pubertad ya que la menor insulinosensibilidad (calificada como el tercil más bajo de índice de QUICKI de una población de referencia nacional sana que incluye normales y obesos) afectó a 53,2\% de los prepúberes y a $54,6 \%$ de los púberes. De la misma forma, no se observaron diferencias por sexo, ya que $61,9 \%$ de las mujeres y $53,2 \%$ de los varones obesos tuvieron un índice de QUICKI igual 0 inferior al tercil más bajo de la población de referencia. El porcentaje GCT se asoció significativamente $(p<0,00)$ con la sensibilidad insulínica basal tanto en prepúberes como en púberes (Figuras 1 y 2). El riesgo de presentar una baja sensibilidad insulínica fue 2,43 veces mayor en los prepúberes con un porcentaje de grasa corporal $\geq 33 \%$ y 2,92 veces mayor en un púber con un porcentaje GCT $\geq 37 \%$.

\section{Discusión}

Los resultados de este estudio confirman la asociación de la resistencia insulínica con el exceso de grasa corporal y con su depósito troncal, tanto en prepúberes como en púberes. Los seguimientos longitudinales demuestran que la cantidad y distri- 


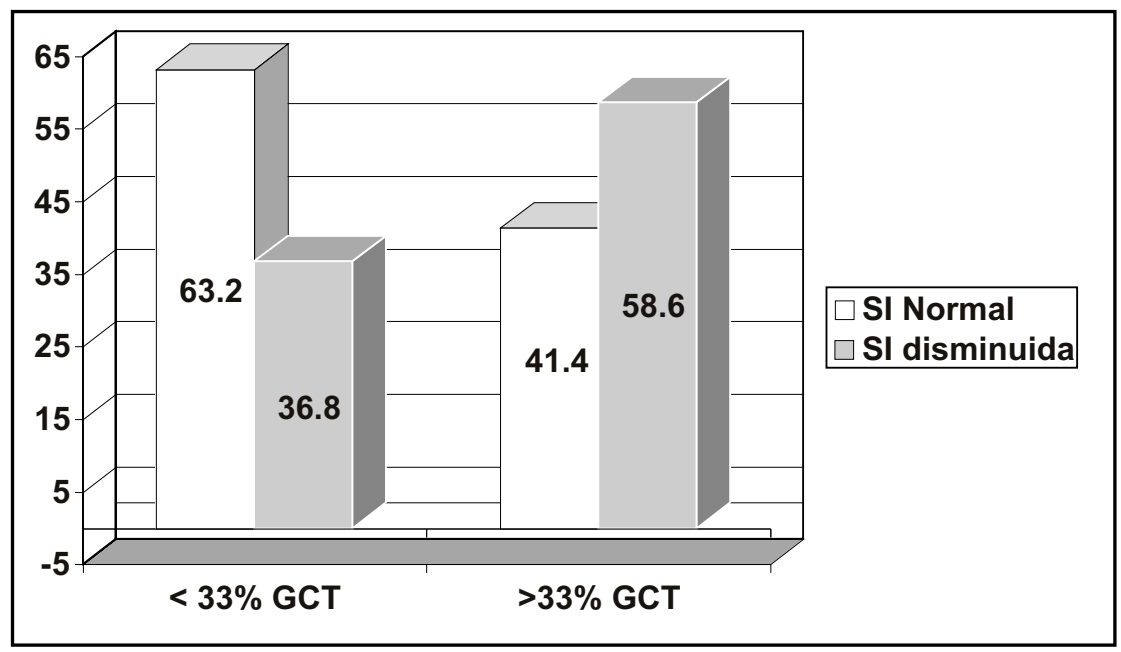

FiguRa 1. Sensibilidad insulínica (SI) en prepúberes según $\% \mathrm{GCT}$.

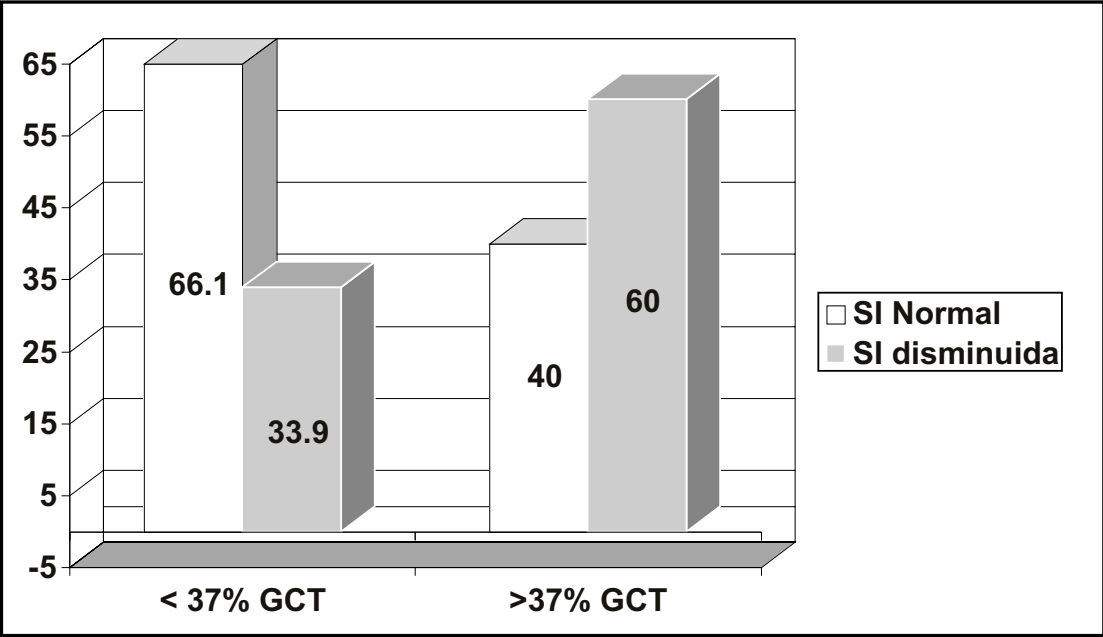

FiguRa 2. Sensibilidad insulínica (SI) en púberes según \% GCT.

bución de la grasa corporal en población infantil determina el daño metabólico, asociándose a un mayor riesgo de enfermedad cardiovascular, dislipidemia y diabetes tipo 2 en la vida adulta ${ }^{24-26}$. En este estudio, el riesgo de una menor sensibilidad insulínica se duplicó cuando el porcentaje GCT excedió $33 \%$ en los prepúberes y se triplicó cuando el porcentaje GCT excedió 37\% en los púberes. En los prepúberes, el punto de corte es coincidente con lo encontrado por un estudio en prepúberes americanos, donde un porcentaje CGT $\geq 33 \%$ evaluado por metodología de DEXA, aumentó significativamente el riesgo (11 veces) de alterar el perfil de insulina y lípidos y la presión $\operatorname{arterial}^{27}$. Otros 2 estudios, uno en población americana y la otra australiana, mostraron que un porcentaje GCT $\geq 30 \%$ en las mujeres y $25 \%$ en los varones, medidos con metodología clínica de sumatoria de pliegues, similar a lo de este estudio, duplica el riesgo de hipertensión arterial, dislipidemia y $\mathrm{R}^{28,29}$. Aun cuando el IMC y el PC mostraron una buena asociación tanto en prepúberes como en púberes con la sensibilidad insulínica basal; en los prepúberes el IMC no muestra diferencias al comparar obesos con sensibilidad normal y disminuida. El PC en cambio, fue un indicador adecuado para diferenciar entre ambas situaciones. Múltiples investigadores han demostrado que el perímetro de cintura sería el indicador antropométrico más sensible para diagnosticar 
la insulinorresistencia en los niños ${ }^{30-32}$. El índice cintura/cadera no se incluyó como variable antropométrica, porque a diferencia de lo observado en población adulta, no mostró diferencias entre insulinosensibles e insulinorresistentes, lo que coincide con otras publicaciones que muestran también al IMC como un indicador de baja sensibilidad en los prepúberes ${ }^{30-32}$.

Si bien en población adulta, el diagnóstico del síndrome metabólico no incluye los niveles de insulina, la RI es el elemento clave en el síndrome metabólico asociado a la obesidad del niño y suele estar presente por muchos años antes que aparezcan otras anormalidades como la intolerancia a la glucosa, la dislipidemia, la HA, la DM2 y las $\mathrm{ECVI}^{33,34}$. No hay consenso en el diagnóstico del síndrome metabólico en los niños y en la mayoría de los casos están basados en criterios estadísticos ${ }^{30-32}$. Algunos autores utilizan la insulinemia basal $(\geq 12 \mathrm{uUI} / \mathrm{ml} 0>$ p 75 de la población de referencia) asociada a un perfil lipídico aterogénico $\mathrm{y}$, últimamente, se han sugerido 2 fenotipos para el diagnóstico en la población infantil que están basados en modificaciones a los 5 criterios de la ATP III del adulto (IMC >p97, presión arterial $>$ p95, triglicéridos $>$ p95, Col-HDL $<p 5$, glicemia basal $>110 \mathrm{mg} / \mathrm{dl}$ y el otro cambia el IMC por el perímetro de cintura, ya que éste tendría mayor sensibilidad para diagnosticar la insulinorresistencia) ${ }^{24,32,35}$. El clamp euglicémico hiperinsulinémico es el indicador más sensible para medir la insulinorresistencia ya que evalúa la captación de glucosa mediada por insulina, sin embargo, su aplicación está limitada por el alto costo y complejidad ${ }^{36}$. En la práctica clínica se utilizan varios indicadores que miden el producto insulina-glicemia basal para calificar la sensibilidad insulínica, pero en los niños, no existe consenso cuál sería el mejor indicador ni el punto de corte para calificar la insulinorresistencia $20-22,37$. Por ese motivo, en este estudio, la insulinosensibilidad fue evaluada por los índices de QUICK y HOMA y consideramos como punto de corte, para definir la menor insulinosensibilidad, el índice de QUICKI equivalente al tercil más bajo en una muestra de 225 niños sanos chilenos ${ }^{23}$. El índice de QUICKI tiene una buena correlación con el clamp euglicémico hiperinsulinémico y tiene la ventaja que predice el riesgo de presentar una DM 2 mejor que el HOMA y la insulina de ayuno, lo que permite un diagnóstico más biológico de esta condición ${ }^{20,21}$. Vanhala y cols determinaron en población adulta, que los sujetos con un índice de QUICKI $\leq 0,32$ tenían un alto riesgo de presentar una diabetes tipo 2 después de 5 años, al compararlos con sujetos con índices mayores ${ }^{21}$. El tercil más bajo del índice de QUICKI de la población que utilizamos como referente para los púberes, coincidió con el valor $\leq 0,32$ descrito por Vanhala y cols como punto de corte de riesgo biológico ${ }^{23}$.

Los obesos con menor sensibilidad insulínica tuvieron un perfil lipídico aterogénico (menor colHDL y mayor triglicéridos y relación CT/col-HDL) y un insuficiente control del metabolismo glucídico, independiente del grado de pubertad alcanzado. Ambos trastornos que determinan un mayor riesgo cardiovascular, se explican porque existiría, por un lado, una mayor secreción de insulina para compensar la menor captación y transporte de glucosa $\mathrm{y}$, por otro, una menor capacidad de suprimir la lipólisis y de depositar los ácidos grasos en el tejido graso, con la consecuente mayor movilización grasa que se deposita en tejidos como el músculo e hígado aumentando aún más la $\mathrm{R}^{4-6}$. Los mayores niveles de glicemia tanto basal como postglucosa en presencia de un hiperinsulinismo marcado, observada en los obesos con sensibilidad insulínica disminuida, sugieren un deterioro en la capacidad de mantener el control glucídico, lo que podría interpretarse como una etapa temprana de intolerancia a la glucosa. Los estudios longitudinales han demostrado que la resistencia periférica a la insulina es la anormalidad primaria que precede a la diabetes y que el hiperinsulinismo sería un mecanismo compensatorio para vencer esta resistencia y regular el metabolismo glucídico en niveles normales. Con el tiempo, el hiperinsulinismo no logra mantener una glicemia normal (etapa de intolerancia a la glucosa) y la hiperglicemia crónica termina dañando (glucotoxicidad) las células beta del páncreas, lo que lleva a un déficit en la secreción de insulina y al comienzo de la enfermedad ${ }^{33}$.

El síndrome metabólico incluye la hipertensión arterial explicada, entre otros, por la sobre respuesta a las concentraciones suprafisiológicas de insulina de los tejidos que no son insulinorresistentes, como el sistema nervioso simpático y por el daño en la función endotelial ${ }^{17,34,38,39}$. En este estudio sin embargo, la presión arterial se mostró 
más asociada a la obesidad que a la resistencia insulínica. Los obesos muestran promedios de presiones arteriales significativamente más elevados que los eutróficos, no observándose diferencias entre los obesos según el grado de insulinosensibilidad. Es posible que la HA sea una manifestación más avanzada de daño endotelial y requiera de un mayor tiempo de exposición al hiperinsulinismo.

La resistencia insulínica no se asoció con la pubertad ni con el sexo femenino, lo que no coincidiría con lo publicado $37,40-42$. Durante la pubertad, la sensibilidad insulínica disminuye casi $30 \%$, especialmente en las mujeres, con el consecuente aumento en la secreción de insulina, sugiriéndose una asociación con el aumento de hormonas de contrarregulación (hormona de crecimiento, IGF-1 e IGF-BP3) ${ }^{40-42}$. Los hallazgos antes descritos corresponderían a "un hiperinsulinismo fisiológico" compensatorio y no podrían

\section{REFERENCIAS}

1. Fagot Campagna A, Pettit DJ, Engaslgau MM, Burrows NR, Geis LS. Type 2 diabetes among North American children and adolescents: an epidemiologic review and a public health perspective. J Pediatr 2000; 136: 664-72.

2. DiETZ WH. Overweight and precursors of type 2 diabetes mellitus in children and adolescents. J Pediatrics 2001; 138: 453-4.

3. CABALERO EA. Endothelial dysfunction in obesity and insulin resistance: a road to diabetes and heart disease. Obesity Res 2003; 11: 1278-89.

4. Keley DE, Wiwams KV, Price JC, McKolanis TM, Goodpaster BH, THAete L Plasma fatty acids, adiposity and variance of skeletal muscle insulin resistance in diabetes type 2 . JCEM 2001; 86: 5412-9.

5. Frayn KN. Adipose tissue and insulin resistance syndrome. Proc Nutr Soc 2001; 60: 375-80.

6. KAHN BB, FLIER JS. Obesity and insulin resistance. J Clin Invest 2000; 106: 473-81.

7. Goran MI, Geoff D, Ball C, Cruz ML. Obesity and risk of type 2 diabetes and cardiovascular disease in children and adolescents. JCEM 2003; 88: 141727. considerarse como un real trastorno metabólico. En el caso de este estudio, se definió la resistencia insulínica utilizando como referente una población de niños normales del mismo sexo y grado de pubertad, lo que permitió considerar las diferencias propias del sexo y del grado de pubertad alcanzado.

Los resultados encontrados demuestran que la sensibilidad insulínica se asocia a la cantidad y distribución de la grasa corporal y a un perfil lipídico de riesgo cardiovascular, desde edades tempranas. Tanto en prepúberes y púberes, la menor sensibilidad insulínica se asoció a un perfil lipídico más aterogénico y a mayores niveles de glicemia tanto basal como postestímulo. La distribución centrípeta y la cantidad de grasa corporal (33\% en los prepúberes y $37 \%$ en los púberes), serían factores asociados a un mayor riesgo de expresar una resistencia insulínica.

8. Must A, Jacques P, Dallal G, Bajema C, Dietz W. Long-term morbidity and mortality of overweight adolescents. A follow-up of the Harvard Growth Study. N Engl J Med 1992; 327: 1350-5.

9. DiETZ W. Health consequences of obesity in youth: Chilhood predictors of adult disease. Pediatrics 1998; 108 (3 II): 518S-525S.

10. Freedman D, Kettel-Khan L, Dietz W, SRinivasan S, BERENSON G. Relationship of childhood obesity to coronary heart disease risk factors in adulthood. The Bogalusa Heart Study. Pediatrics 2001; 108: 712-8.

11. Limola $S$, Nyomba BL, SAad MF, Ferraro $R$, Castillo C, Bennett P et al. Exaggerated early insulin release and insulin resistance in a diabetes prone population: A metabolic comparison of Pima Indians and Caucasians. JCEM 1991; 73: 866-76.

12. Lumola S, Mott DM, Zawadzki JK, Young AA, АввотT WGH, KNowler WC ET AL. In vivo insulin action is familial characteristic in nondiabetic Pima Indians. Diabetes 1987; 36: 1329-35.

13. Pinhas-Hamiel O, Dolan LM, Daniels SR, Standford D, Khoury PR, Zeltier P. Increased incidence of non-insulin dependent diabetes mellitus among adolescents. J Pediatr 1996; 128: 608-13. 
14. Rocco P, Morales C, Moraga M, Miquel JF, Nervi F, Lop E ET AL. Composición genética de la población chilena. Distribución de polimorfismos de $\mathrm{ADN}$ mitocondrial en grupos originarios y en la población mixta de Santiago. Rev Méd Chile 2002; 130: 125-31.

15. Burrows R, Gattas V, Leiva L, Barrera G, Burgueño M. Características biológicas, familiares y metabólicas de la obesidad infantil y juvenil. Rev Méd Chile 2001; 129: 1155-62.

16. Norma Técnica de Evaluación Nutricional del Niño de 6 a 18 años. Unidad de Nutrición del MINSAL Consejo Asesor de Nutrición. Grupo de Expertos. Rev Chil Nutr 2004; 31: 128-37.

17. Wong W, Stuff J, Butte N, Smith E, Eus K. Estimating body fat in African American and white adolescent girls: a comparison of skinfoldthickness equations with a 4 compartment criterion model. Am J Clin Nutr 2000; 72: 348-54.

18. Weststrate J, Deurenberg P. Body composition in children: proposal for a method for calculating body fat percentage from total body density or skinfold-thickness measurements. Am J Clin Nutr 1989; 50: 1104-15.

19. National Health, Lung and Blood Institute. Report of second task force on blood pressure in children, 1987. Pediatrics 1987; 79: 1-2.

20. Perseghin G, Caumo H, Caloni M, Testolin G, Luzi L. Incorporation of the fasting plasma FFA concentration into QUICKI improve its association with insulin sensitivity in nonobese individuals. JCEM 2001; 86: 4776-81.

21. Vanhala P, Vanhala M, Kumpusalo E, KeinanenKIUKAANNIEMI S. The quantitative insulin sensitivity check index >QUICKI predicts the onset of diabetes better than fasting plasma insulin in obese subjects; a 5 years follow-up study. JCEM 2002; 87: 5834-7.

22. Matthews DR, Hosker JP, Rudenski AS, Naylor BA, Treacher DF, Turner RC. Homeostasis model assessment insulinresistance and beta cell function from fasting glucose and insulin concentrations in man. Diabetologia 28: 412-9. año??

23. Burrows R, Leiva L, Burgueño M, Maggi A, Jiadrosic V, Díaz E. Sensibilidad insulínica en población normal de 6 a 15 años evaluada a través de diferentes indicadores clínicos. Rev Chil Pediatr 2004; 75(6): 561-641.
24. Cook S, Weitzman M, Auinger P, Nguyen M, Dietz W. Prevalence of metabolic syndrome phenotype in adolescents. Arch Ped Adol Med 2003; 157: 821-7.

25. Asayama K, Hayashibe H, Dobashi K, Uchida N, KaWada Y, Nakazawa S. Relationships between biochemical abnormalities and anthropometric indices of overweight, adiposity and body fat distribution in Japanese elementary school children. In J Obesity 1995; 19: 254-9.

26. Wiwams D, Going S, Lohman T. Body fastness and risk for elevated blood pressure, total cholesterol and serum lipoprotein rations in children and adolescents. Am J Public Health 1992; 82: 358-63.

27. Higgins P, Gower B, Hunter G, Goran M. Defining health related obesity in prepubertal children. Obes Res 2001; 9: 233-40.

28. Dwyer T, BuZzard CL. Defining obesity in children by biological endpoint rather than population distribution. Int J Obes 1996; 20: 472-80.

29. Mamalakis G, Kafatos A, Manios Y, Kalogeropoulos $\mathrm{N}$, Andrikopoulos N. Abdominal vs buttock adipose fat: relationships with childrens serum lipid levels. Eur J Clin Nutr 2002; 56: 1081-6.

30. Moreno LA, Pineda I, Rodríguez G, Fieta J, Sarría A, Bueno M. Waist circumference for the screening of metabolic syndrome in children. Acta Paediatr 2002; 91: 1307-12.

31. Savva SC, Tornaritis M, Savva ME, Kouridis $Y$, Panagi A, Georgiuo C et al. Waist circumference and waist-to-height ratio are better predictors of cardiovascular disease risk factors in children than body mass index. Int J Obe 2000; 24: 1453-8.

32. Maffeis C, Pietrobe山u A, Grezzani A, Provera S, TAтó L. Waist circumference and cardiovascular risk factors in prepubertal children. Obesity Res 2001; 9: 179-87.

33. Martin BC, Warram JH, Krolewski AS, Bergman RN, SOELDNER JS, KaHN CR. Role of glucose and insulin resistance in development of type 2 diabetes mellitus: results of 25 years follow-up study. Lancet 1992; 340: 925-9.

34. GoLdSTEIN BJ. Insulinresistance as the core defect in type 2 diabetes mellitus. Am J Cardiol 2002; 90: 3G-10G.

35. Weiss R, Dziura J, Burgert TS, Tamborlane WV, TAKSALI SE, YeCKel CW ET AL. Obesity and metabolic syndrome in children and adolescents. $\mathrm{N}$ Engl J Med 2004; 350: 2362-74. 
36. Weiss R, Dufour $S$, Taksali $S$, Tamborlane W, Banadonna R, Aluen K et al. Prediabetes in obese youth: a syndrome of impaired glucose tolerance, severe insulin resistance and altered myocellular and abdominal fat partitioning. Lancet 2003; 362: 951-7.

37. Hoffman RP, Vicin P, Sivitz WI, Coвeшi C. Pubertal adolescent male-female differences in insulin sensitivity and glucose effectiveness determined by the one compartment minimal model. Pediatric Research 2000; 48: 384-8.

38. Fisch SR, Tamborlane TB, Banyas B, Aluen K. Prevalence of impaired glucose tolerance among children and adolescent with marked obesity. N Engl J Med 2002; 346: 802-10.
39. GINSBERG HN. Insulinresistance and cardiovascular disease. J Clin Invest 2000; 106: 456-8.

40. Travers SH, JefFers BW, Bloch CA, HiL JO, Eckel $\mathrm{RH}$. Gender and Tanner stage differences in body composition and insulin sensitivity in early pubertal children. JCEM 1995; 80: 172-8.

41. Morán A, Jacobs D, Steinberger J. Association between the insuline resistance of puberty and insuline-like-growth factor-I/Growth hormone axis. JCEM 2002; 87: 4817-20.

42. Silfen M, Manibo AM, Ferin M, McMahon DJ, Levine L, OBERFIELD S. Elevated free IGF-1 levels in prepubertal hispanic girls with premature adrenarche, relationship with hyperandrogenism and insulin sensitivity. JCEM 2002; 87: 398-403. 